Voix et Images

\title{
Petit Manuel des études littéraires. Pour une science générale de la littérature, de Guy Laflèche
}

\section{Jean Fisette}

Volume 3, numéro 1, septembre 1977

Nicole Brossard

URI : https://id.erudit.org/iderudit/200096ar

DOI : https://doi.org/10.7202/200096ar

Aller au sommaire du numéro

Éditeur(s)

Les Presses de l'Université du Québec

ISSN

0318-9201 (imprimé)

1705-933X (numérique)

Découvrir la revue

Citer cet article

Fisette, J. (1977). Petit Manuel des études littéraires. Pour une science générale de la littérature, de Guy Laflèche. Voix et Images, 3(1), 151-153.

https://doi.org/10.7202/200096ar d'utilisation que vous pouvez consulter en ligne. 
Petit Manuel des études littéraires. Pour une science générale de la litterature, de Guy Laflèche

Voilà un ouvrage paradoxal, difficile sinon impossible à situer; tout est question de “ton" a-t-on déjà dit! Le mouvement de l'écriture passe 
de l'ironie à la polémique qui avoue son innocence, c'est à dire une ironisation de l'ironie; c'est peut-être compliqué, mais c'est exactement la fonction introductive de la première phrase, d'ailleurs reprise à l'endos du livre: «Cet essai, lit-on, n'est pas polémique et on peut espérer qu'il ne choquera que l'ignorance et l'incompétence, surtout de celles qui sont de bonne volonté..."

L'auteur, dans maints passages, emprunte volontiers le ton familier, se permettant de dénigrer ce qui, à quelques pages de distance, à été posé comme important, sérieux, engagé, significatif. Alors, où se situer? Comment percevoir le livre, quelle disposition d'esprit permettra de le lire? Ou encore: à qui s'adresse le livre? aux étudiants en littérature? aux professeurs et étudiants savamment diplômés (ou en voie de l'être)? S'agit-il d'un ironique «Petit manuel » ou de prolégomènes à une "science générale"? Chacun peut y trouver son compte, comme éclaircissement théorique ou comme pilule à avaler? Dans notre société de surdiplômation, le seul prestige à tirer d'un acquis de connaissances (diplômes), m'a-t-on déjà laissé entendre, c'est un "droit de dire ce que l'on veut, à qui l'on veut, quand on le veut bien»! Voilà la leçon magistrale que G. Laflèche semble avoir, sinon comprise, du moins bien assimilée...

Voilà pour le ton qui est peut-être tout le livre. Le prétexte à ce jeu pour intellectuels, c'est de couvrir à peu près tout le champ théorique, technique, pratique, qui entoure la production du livre, si ce n'est le simple détail de l'écriture du texte. Au fil des chapitres, on passe de la critique littéraire à la description analytique du texte, au travail d'élaboration théorique sous-tendant cette «science générale de la littérature»; du modèle théorique structural au champ de la sémiotique, en ménageant aussi une place à la lecture thématique des œuvres; littérature comparée, géographie de la littérature, dimension temporelle et histoire de la littérature (il y a quelque chose du fascicule de présentation des programmes universitaires dans cette nomenclature); et, pour finir: le domaine de la philologie encadrant les questions de bibliographie, édition, la critique (encore!), les techniques littéraires (encore!); et nous voici au dessert présenté en trois plats: l'en deçà et l'au-delà philosophiques, caractérologie (vous avez bien lu) du critique littéraire et de l'analyste, matérialisme et désacralisation de la littérature. La fine, pour terminer ce repas gastronomique, nous conduira, croit-on, à la quintessence de la scientificité de la littérarité; mais non, elle s'intitule: fiction et réalité.

Tout ceci en quelque cent pages (de gros caractères)! II n'y a pas de gastronomie mais, plus bêtement, un copieux repas!

II y a vraiment lieu de se poser de sérieuses questions, de douter de l'efficacité du traitement des sujets “abordés».

Si le modèle linguistique emprunté à Hjelmslev (la quadripartition de la stratification du signe linguistique) peut s'avérer utile, voire efficace, à un certain stade de la disposition des perspectives analytiques, tout ra- 
mener à ce schéma, c'est de la simplification abusive - si ce n'est que dans ces conditions, toutes ces avenues peuvent se tasser dans un même tableau, satisfaction béate pour l'œil qui se prend pour l'esprit. Par exemple, je ne comprends vraiment pas que l'on puisse, en superposant différentes grilles, poser en équivalence syntaxe et forme de l'expression; histoire et substance du contenu; récit et forme du contenu; lexique et substance de l'expression. Je fais remarquer que le schéma (dont je vous fais grâce) s'intitule: «Représentation simplifiée du texte littéraire " (p. 46). Le terme "simplifiée " connote certainement chez Laflèche, une rhétorique de l'euphémisme.

Mais là n'est pas le pire; je cite, pour exemple, cette perle:

La seule application qu'on en [analyse sémique] connaît actuellement, et qu'on veut appeler la sémiotique, tente de dépasser le niveau de la phrase, où se limite la description linguistique, par la notion d'ISOTOPIE qui pourtant la respecte. On se contentera de remarquer ici que ce qu'on nomme actuellement isotopie est une rationalisation ou une description de ce qu'on appelait vaguement jusqu'ici le "contexte" et que son étude permettra peut-être de faire le lien entre l'analyse sémantique et celle des contenus littéraires beaucoup plus larges. (p. 40)

C'est devant un tel passage que je regrette que les marges du livre soient si étroites: ne pouvant y inscrire mes réactions, je les écris ici:

1. La notion d'isotopie relève strictement de la sémantique; c'est une appartenance commune de sèmes, permettant de formaliser le(s) plans(s) d'homogénisation d'une série d'énoncés. L'isotopien n'est quand même pas un simple substitut moderne de la traditionnelle "description de contexte».

2. La sémiotique est un champ de recherche (pluri-disciplinaire) se donnant comme objet de décrire les mécanismes de production de sens. Or, il se produit que la sémiotique se fonde plus sur le signifiant (la linguistique proprement dite) que sur le signifié (la sémantique).

3. Jusqu'à maintenant, la notion d'isotopie s'est avérée l'instrument le plus efficace pour rendre compte d'une organisation (ou structuration) transphrastique, quoique cet instrument se révele très faible en raison de sa relation de dépendance à la pratique de la lecture (impliquant la subjectivité du lecteur). Une isotopie, il ne faudrait pas l'oublier, est toujours une "isotopie de lecture".

Mais à quoi sert un tel commentaire: si l'on a le malheur de prendre le texte au sérieux, on en arrive à la conclusion qu'il serait à réécrire... Et encore, je ne crois pas que soit encore arrivée l'heure des vastes bilans.

Ce qui me ramène à mon point de départ: que vise ce livre? Qui vise-t-il? On ne peut que regretter que l'éditeur ne se soit pas posé ces questions... à moins qu'il ait un goût irrésistible pour les petits manuels... 